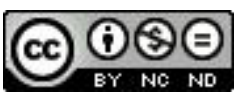

$\overline{\text { ARTIGOS }}$

\title{
A Gênese Instrumental na Interação com o GeoGebra: uma proposta para a formação continuada de professores de Matemática
}

\section{The Instrumental Genesis and its Interaction with GeoGebra: a proposal for continuing education for mathematics teachers}

\author{
Celina Aparecida Almeida Pereira Abar* \\ Sergio Vicente Alencar**
}

\begin{abstract}
Resumo
Este trabalho apresenta resultados de uma pesquisa de Mestrado Profissional em Ensino de Matemática do Programa de Estudos Pós-Graduados em Educação Matemática da Pontifícia Universidade Católica de São Paulo (PUC-SP), desenvolvida no Grupo de Pesquisa Tecnologias e Meios de Expressão em Matemática. A pesquisa teve como objetivo o desenvolvimento de uma oficina de formação continuada, com o uso do GeoGebra, para professores de Matemática da escola básica. O referencial teórico subjacente é a Abordagem Instrumental de Pierre Rabardel, com foco na Gênese Instrumental, que estuda a transformação de um artefato em instrumento. A metodologia utilizada é o Design Experiments, escolhida por permitir a realização de uma avaliação formativa para executar e refinar projetos educacionais. Por meio da analise dos resultados, verificou-se que a maioria dos professores conseguiu alcançar o processo de instrumentalização e que o ambiente dinâmico propiciado pelo software GeoGebra colaborou para a instrumentação.
\end{abstract}

\footnotetext{
* Doutora em Matemática pela Pontifícia Universidade Católica de São Paulo (PUC/SP). Professora do Programa de Estudos Pós-Graduados em Educação Matemática da pela Pontifícia Universidade Católica de São Paulo (PUC/SP). Endereço para correspondência: Rua Marquês de Paranaguá, 111, Consolação, CEP: 01303-050, São Paulo, SP, Brasil. E-mail: abarcaap@pucsp.br.

*** Mestre em Ensino da Matemática pela Pontifícia Universidade Católica de São Paulo (PUC/SP). Professor de Matemática e Física do Colégio Piaget. Endereço para correspondência: Rua Epaminondas Melo do Amaral, 99/156, Imirim, CEP: 02022-120, São Paulo, SP, Brasil. E-mail: sergiomatematica@yahoo.com.br.
} 
Palavras-chave: Educação Matemática. Gênese Instrumental. GeoGebra. Tecnologias da Informação e Comunicação.

\begin{abstract}
This paper presents the results of research carried out as part of the Professional Masters in Mathematics Teaching of the Post-Graduate Studies Program in Mathematics Education of the Pontifical Catholic University of Sao Paulo (PUC-SP), developed by the Research Group Technologies and Means of Expression in Mathematics. The research aimed to develop a continuing education workshop, with the use of GeoGebra, for primary school mathematics teachers. The theoretical reference was Pierre Rabardel's instrumental approach, focused on the Instrumental Genesis, which examines the transformation of an artefact into an instrument. The methodology used was Design Experiments that allows a formative evaluation to implement and refine educational projects. Through the analysis of the results, it was found that most teachers managed the instrumentalization process and that the dynamic environment offered by the software GeoGebra helped the instrumentation.
\end{abstract}

Keywords: Mathematics Education. Instrumental Genesis. GeoGebra. Information and Communication Technologies.

\title{
1 Introdução
}

Um desafio para o processo de ensino e aprendizagem de Matemática é a inserção de diferentes recursos na prática pedagógica do professor. Um desses recursos é o tecnológico, que, de acordo com os Parâmetros Curriculares Nacionais:

[...] é um instrumento capaz de aumentar a motivação dos alunos, se a sua utilização estiver inserida num ambiente de aprendizagem desafiador. Não é por si só um elemento motivador. Se a proposta de trabalho não for interessante os alunos rapidamente perdem a motivação (BRASIL, 1997, p. 57).

Desse modo, esta pesquisa teve como objetivo desenvolver uma oficina de formação continuada ${ }^{1}$, com o uso do GeoGebra, para cinquenta e cinco professores de Matemática da escola básica, tendo como referência a Abordagem Instrumental de Pierre Rabardel (1995). Conjeturou-se que, ao seguir os parâmetros delineados durante a execução da oficina, os professores poderiam

\footnotetext{
${ }^{1}$ Todas as seis lições da oficina estão disponíveis no endereço: <http://www.pucsp.br/tecmem/ sergio_alencar.pdf>. Acesso em: 04 mar. 2013.
} 
aprimorar suas interpretações ou modos de pensar a Matemática por meio da tecnologia.

Para alcançar o objetivo, formulou-se a seguinte questão de pesquisa: quais orientações são necessárias para que uma oficina de GeoGebra possibilite que professores de Matemática da escola básica possam elaborar estratégias próprias para sua prática com o uso desse software?

A proposta da oficina foi estruturada de acordo com a Gênese Instrumental de Rabardel, porque consideramos este aporte teórico uma importante orientação para atender nossos objetivos. Segundo essa teoria, não é necessária apenas a inclusão de usuários em atividades que utilizam a tecnologia, caracterizada por Rabardel (1995) como um artefato que pode ser transformado em um instrumento. Também, devem-se considerar os processos pelos quais os usuários transformam o artefato em instrumento, denominada por Rabardel de Gênese Instrumental.

Laborde e Sträßer (2010, p.12), nas considerações finais do artigo, afirmam que:

Parece óbvio que uma mera análise dos artefatos (computadores, software, tecnologia de comunicação) não é suficiente para permitir que essa tecnologia seja usada no ensino e no aprendizado da matemática. Os "estudos de usuários" (muitas vezes mencionados em informática) são um pré-requisito inevitável para a implementação de novas tecnologias na aula de matemática. Para fazer essa afirmação com a terminologia de um dos referenciais teóricos amplamente utilizados na didática da matemática (ver Rabardel 1995): a análise do artefato é um pressuposto insuficiente para introduzir e compreender o seu uso. Só uma análise do instrumento, ou seja, da interação do artefato e dos métodos de utilização de seus usuários (professores e alunos), e da análise da sua "gênese instrumental" irá ajudar na implementação de computadores, software e tecnologia de comunicação na aula de matemática (tradução própria) $)^{2}$.

\footnotetext{
${ }^{2}$ Traduzido de: It seems obvious that a mere analysis of the artifacts (computers, software, communication technology) is not sufficient to make this technology to be used in teaching and learning mathematics. "User studies" (often referred to in informatics) are an unavoidable prerequisite for the implementation of new technology in the mathematics classroom. To state it in the terminology of one of the theoretical frameworks widely used in didactics of mathematics (see Rabardel 1995): the analysis of the artifact is an insufficient presupposition to introduce and understand its use. Only an analysis of the instrument, i.e., the interaction of the artifact and the utilization schemes of its users (teachers and students), the analysis of its "instrumental genesis" will help in the implementation of computers, software and communication technology in the mathematics classroom.
} 
Desse modo, transformar o software GeoGebra em um instrumento é importante, pois, nessa evolução, ocorrem a reorganização e a modificação dos esquemas de utilização, fatos que permitem a estruturação da ação do professor, colaborando para sua formação e aprimoramento de conceitos matemáticos.

A proposta foi que o professor utilizasse o software GeoGebra não apenas como mais um recurso tecnológico, mas, sim, como um recurso que colaborasse no desenvolvimento de conceitos matemáticos, uma vez que, por si só, o software não faz Matemática. Dessa forma, esta pesquisa poderá colaborar para a inserção da tecnologia na prática docente, aprimorando os estudos e as análises no que diz respeito à tecnologia no contexto da educação matemática.

Para se chegar ao produto final com seis lições, três oficinas com o uso do GeoGebra foram realizadas a um contingente de cinquenta e cinco professores de Matemática com suporte na metodologia de pesquisa Design Experiments que, de acordo com Doerr e Wood (2006), permite a realização de uma avaliação formativa para testar e aperfeiçoar modelos educacionais baseados em princípios derivados de investigação prévia.

\section{Abordagem instrumental de Rabardel}

A Abordagem Instrumental de Rabardel (1995) se apoia na teoria da ergonomia cognitiva, referente aos processos mentais (percepção, memória, raciocínio etc.) que afetam as interações entre seres humanos e outros elementos de um sistema, sendo a interação homem-computador um exemplo disso.

Rabardel (1995) descreve as relações que existem entre o sujeito, a ferramenta (artefato) e os esquemas de utilização. Sujeito: indivíduo ou grupo de indivíduos que desenvolvem a ação ou são escolhidos para o estudo. Artefato: dispositivo que pode ser material (lápis, computador etc.) ou simbólico (uma figura, um gráfico etc.). Esquemas de utilização: Rabardel (1995) utiliza esse termo que, de acordo com Vergnaud (1996), “[...] é uma organização invariante de comportamentos para classes de situações". É necessário procurar nos esquemas os elementos cognitivos que permitem que a ação do sujeito seja operatória.

A Abordagem Instrumental estuda os aspectos próprios que existem no artefato e no instrumento, e processos que envolvem a transformação progressiva do artefato em instrumento, denominada de Gênese Instrumental. Para Verillon e Rabardel (1995 apud SALAZAR, 2009), esse processo busca a integração entre as características dos artefatos (potencialidades e limitações) e as atividades 
do sujeito - seus conhecimentos e métodos de trabalho.

O foco de interesse de Rabardel (1995) é a transformação do uso do artefato em um instrumento, propondo, então, o modelo de situações de utilização de um instrumento, composto por: sujeito: usuário, operador, trabalhador etc. É ele que dirige a ação psíquica sobre o objeto; instrumento: ferramenta, máquina, produto etc. É o mediador entre o sujeito e o objeto; objeto: material, real, objeto da atividade, objeto de trabalho ou outros sujeitos. É sobre ele que a ação é dirigida.

Rabardel (1995) propõe o modelo SAI (Situações de Atividades Instrumentais), apresentando as relações entre o sujeito e o objeto mediado pelo instrumento. Para Rabardel (1995), o modelo SAI (Figura 1) evidencia as várias interações que intervêm nas atividades instrumentais: sujeito-objeto [S-O], sujeitoinstrumento [S-i], instrumento-objeto [i-O] e sujeito-objeto mediada pelo instrumento [S(i)-O], que se desenvolvem em um ambiente formado pelo conjunto de condições que o sujeito deve levar em conta para realizar sua atividade.

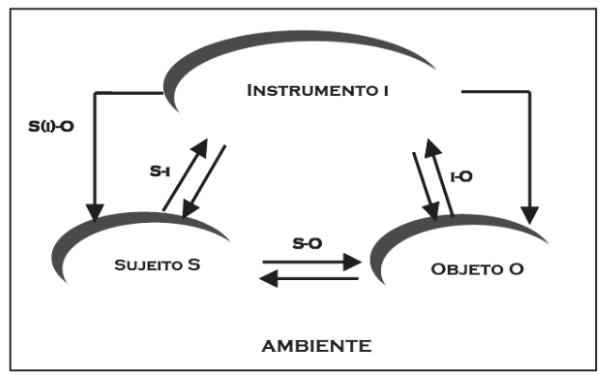

Figura 1 - Modelo de Situações de Atividades Instrumentais Fonte: Rabardel (1995, p. 65).

O modelo SAI pode ser uma ferramenta para examinar, detalhadamente, o uso de instrumentos em uma tarefa. O instrumento como mediador possui a orientação de Objeto-sujeito (é o meio que permite o conhecimento do objeto) e de Sujeito-objeto (é o meio da ação transformadora dirigida sobre o objeto).

Para Rabardel (1995), o instrumento é uma entidade mista com dois componentes: o artefato, produzido para o sujeito; e os esquemas de utilização associados, que são resultados de uma construção do próprio sujeito ou de uma apropriação de esquemas de utilização já existentes. O instrumento como artefato é constituído no(s) uso(s) que o sujeito faz dele. Dessa forma, os usos do artefato dependem, também, das necessidades e objetivos do usuário. 
Rabardel (1995) justifica que os esquemas são formados pelo componente psicológico do instrumento que organiza a atividade (novos esquemas, esquemas pessoais ou sociais pré-existentes), assim, os esquemas mediam o sujeito e sua atividade. Rabardel (1995) denomina os esquemas relacionados ao uso do artefato como esquemas de utilização (E.U.), tendo relação com duas dimensões da atividade: atividades relacionadas às tarefas secundárias (são as tarefas relativas à gestão das características e propriedades particulares do artefato funcionamento e manipulação), na qual os esquemas são definidos como esquemas de uso (E.Us.); atividades primárias (orientadas ao objeto da atividade, na qual o artefato é um meio de concretização e de realização), em que os esquemas são definidos como esquemas de ação instrumental (E.A.I.). Um mesmo esquema pode ter um estatuto de E.Us. ou de E.A.I..

Rabardel (1995) destaca que, com base nesses esquemas, surgem os esquemas de atividade coletiva instrumental (E.A.C.I.), pois os sujeitos inseridos em uma atividade coletiva valem-se de esquemas de utilização, que apresentam a coordenação de ações individuais e a integração de seus resultados para atender aos objetivos comuns. Portanto, o coletivo trabalha com um instrumento ou com uma mesma classe de instrumentos, fazendo com que os esquemas de utilização possuam uma dimensão privada e outra social.

Os diferentes tipos de esquemas são mutuamente dependentes. Dessa forma, o instrumento é uma entidade dinâmica, que evolui segundo as situações nas quais a ação do sujeito é engajada.

A composição e a origem de um instrumento, segundo o autor, dependem de seus invariantes: esquemas e artefatos são instrumentalizados (utilizados) pelo sujeito; mas, os esquemas pertencem ao sujeito e são generalizados ou acomodados por ele ao artefato e, às vezes, esquemas novos devem ser construídos. Os processos descritos distinguem-se em termos de instrumentação e instrumentalização, que são denominados, pelo autor, Gênese Instrumental.

Conforme Rabardel (1995), a Gênese Instrumental tem duas dimensões: a instrumentação (orientada para o sujeito): tem relação ao surgimento e evolução de esquemas de utilização e da ação instrumental. Zuchi (2008) caracteriza a instrumentação como um processo pelo qual as especificidades e as potencialidades de um artefato vão condicionar as ações de um sujeito para resolver um dado problema e a instrumentalização (orientada para o artefato): tem relação com o enriquecimento das propriedades do artefato. Zuchi (2008) caracteriza a instrumentalização como um processo pelo qual o sujeito modifica, adapta ou produz novas propriedades, personalizando o artefato de acordo com 
suas demandas. Por exemplo, quando o indivíduo personaliza o computador de acordo com suas necessidades: acessibilidade dos programas, barra de ferramentas, formato de telas, dentre outras.

É importante observar que as duas dimensões do processo de Gênese Instrumental referem-se ao sujeito e ao objeto, mas com orientações diferentes. Assim, ambas contribuem para a evolução do instrumento, para a reorganização e modificação dos esquemas de utilização do sujeito, permitindo a estruturação de sua ação e a participação da formação dos conceitos matemáticos.

No modelo SAI (Figura 1), a instrumentação é a relação entre sujeito e instrumento (S-i) e a instrumentalização é a relação entre sujeito e objeto, mediada pelo instrumento (S(i)-O), assim como a relação entre instrumento e objeto (i$\mathrm{O})$.

Rabardel e Waern (2003) destacam que não é necessária apenas a inclusão de usuários em atividades que utilizam um artefato (computador), mas, também, considerar os processos pelos quais os usuários transformam o artefato em instrumento.

\section{Metodologia}

A metodologia utilizada nesta pesquisa é denominada Design Experiments ou Design Research, que, de acordo com Collins et al. (2004), foi introduzida em 1992, por Ann Brown e Allan Collins. Experimentos utilizando essa metodologia foram desenvolvidos com o objetivo de realizar avaliação formativa para testar e refinar projetos educacionais baseados em princípios derivados de pesquisas anteriores.

Visando à minimização de obstáculos, o Design Experiments permite um progressivo aprimoramento da investigação, pois se aplica uma primeira versão de um projeto para posterior análise, resultando numa revisão baseada nas experiências colhidas e avaliadas.

Dois princípios norteiam o Design Experiments, de acordo com Doerr e Wood (2006). Um deles é o desenvolvimento de um processo ou de um produto aprimorado. No que diz respeito à aprendizagem de docentes, esse trabalho busca aprimorar as interpretações ou modos de pensar que os professores utilizam para dar sentido ao seu ensino e os artefatos e instrumentos que são utilizados em seu trabalho, mais especificamente o software GeoGebra.

O outro princípio destacado por Doerr e Wood (2006) refere-se aos vários ciclos de análise necessários, com a finalidade de aprimorar o produto. 
Isso implica que a coleta e a interpretação dos dados não ocorram ao término da atividade (experimento). No caso deste trabalho, o produto educacional final é uma proposta de oficina para o uso do GeoGebra.

No Design Experiments, três tipos de variáveis dependentes devem ser avaliadas: variáveis de clima (cooperação entre os aprendizes, compromisso, grau de esforço apresentado pelos aprendizes etc.) e avaliada por meio de técnicas de observação - como intervenção na prática ou gravações em vídeo - e notas de campo; variáveis de aprendizagem (conhecimentos, conteúdo, habilidades e disposições) e avaliada, por exemplo, por meio de entrevistas orais, pré-testes, pós-testes e perguntas e respostas curtas; variáveis sistêmicas (alteração, expansão, sustentabilidade, alteração, facilidade de adoção e custos), avaliada por meio de entrevistas estruturadas e pesquisas e/ou por meio de relatos que mostrem as vantagens e dificuldades encontradas pelos professores ao realizar o experimento.

Outras variáveis, denominadas por Collins et al. (2004) como contextuais, podem influenciar o sucesso do experimento na prática. Algumas dessas variáveis são: o ambiente (a inovação só é determinada se experimentada em ambientes distintos), a natureza dos aprendizes (qual é o público alvo?), bem como os recursos necessários e apoio para implementação (técnicos, materiais, administrativos etc.).

As variáveis contextuais e dependentes são inter-relacionadas, afirmam Collins et al. (2004). Assim, mudanças em uma variável têm efeitos em outras no momento da avaliação.

Esses autores justificam o uso do Design Experiments porque ele é contextualizado em situações educativas, mas com um foco em generalizar suas configurações para orientar o processo e o sucesso do experimento. De acordo com os autores, o Design Experiments preenche um vazio na matriz de métodos experimentais que é necessário para melhorar as práticas educacionais.

\section{Procedimentos metodológicos}

Neste trabalho foram realizadas três oficinas, cujos sujeitos participantes da pesquisa eram professores de Matemática da rede estadual de São Paulo, aos sábados, nos laboratórios de informática da Pontifícia Universidade Católica de São Paulo (PUC-SP).

Foi enviado um e-mail às diretorias de ensino, comunicando a realização das oficinas, sendo responsabilidade das diretorias de ensino a divulgação para 
os professores. O professor interessado deveria enviar um e-mail, declarando seu interesse na participação e a seleção dos professores foi por ordem de recebimento desses e-mails.

As vagas para a participação decorreram de acordo com a disponibilidade física dos laboratórios, sendo disponibilizado um computador por participante com o software GeoGebra devidamente instalado. Foram realizadas oficinas com três turmas diferentes.

A primeira oficina, denominada oficina piloto, teve a duração de 8 horas e foi dividida em dois encontros presenciais de 4 horas cada, realizada em dois sábados sucessivos no final do $2^{\circ}$ semestre de 2010 . Foram 27 os professores participantes.

Ao final da oficina piloto, verificou-se que o tempo necessário para a realização não foi suficiente, pois muitos professores realizaram as atividades de forma acelerada, sem o tempo necessário para as reflexões propostas. Além disso, optou-se que, nas próximas oficinas, fosse fortalecida a característica de intervenção do Design Experiments, que, segundo Cobb et al. (2003), possui como intenção a investigação das possibilidades de melhoria educacional. Assim, o autor do trabalho poderia fortalecer sua comunicação com os professores participantes, com maior interação.

A segunda oficina foi programada para ter a duração de 16 horas, sendo dividida em quatro encontros presenciais de 4 horas cada, realizada em quatro sábados consecutivos do início do $1^{\circ}$ semestre de 2011. Foram 13 os professores participantes.

Como o tempo designado para a segunda oficina foi considerado suficiente, variável sistêmica do Design Experiments, na terceira, foi mantida a duração de 16 horas, sendo dividida em quatro encontros presenciais de 4 horas cada, realizada em quatro sábados consecutivos do início do $2^{\circ}$ semestre de 2011. Foram 15 os professores participantes.

\section{As atividades propostas nas oficinas}

A oficina foi dividida em seis lições e composta por atividades. Como já mencionado, essas lições estão disponíveis em <http://www.pucsp.br/tecmem/ sergio_alencar.pdf $>$.

Neste artigo, são apresentadas as duas primeiras lições e analisadas apenas as atividades da Lição I, pois o seu desenvolvimento permitiu uma reflexão importante sobre parte da proposta apresentada. Essa atividade foi estruturada 
da seguinte forma:

Lição 1 - Polígonos e Ângulos:

- Atividade I: Construção de um triângulo e medição da soma dos ângulos internos.

- Atividade II: Construção de um triângulo equilátero.

A escolha do tema triângulo para a primeira lição ocorreu para que os professores pudessem perceber, logo de início, que o software não faz Matemática sozinho, sendo necessária a ação e o conhecimento do usuário.

A discussão sobre a soma dos ângulos internos de um triângulo qualquer é um exemplo disso: apesar de a soma dos ângulos internos dos triângulos construídos pelos usuários ser $180^{\circ} \mathrm{e}$, com a movimentação dos vértices, permitida pelo software, permanecer constante, a simples construção e o dinamismo da manipulação da construção não demonstram, formalmente, que isso é válido para qualquer triângulo, pois os diferentes triângulos obtidos com o software não abrangem todas as infinitas possibilidades de triângulos.

A construção do triângulo equilátero também aborda ofazer Matemática, pois o dinamismo do software GeoGebra permite a alteração de qualquer triângulo equilátero construído por aproximação, ou seja, o usuário clica em três pontos quaisquer da tela que aparentam dar como resultado, quando traçados segmentos de retas, um triângulo equilátero. Para o triângulo permanecer equilátero, independente da movimentação dos vértices, é necessário que o usuário utilize o processo de construção de um triângulo equilátero, que será aprofundado posteriormente, ou seja, são necessários os conhecimentos de conceitos matemáticos para isso.

Lição 2 - Retas Perpendiculares e Paralelas:

- Atividade III: Construção do ponto médio de um segmento de reta.

- Atividade IV: Construção de uma reta perpendicular a uma reta conhecida por meio de um determinado ponto.

- Atividade V: Construção de uma reta paralela a uma reta conhecida por meio de um determinado ponto.

A lição 2 teve como escolha o tema retas perpendiculares e paralelas, pois, por meio de suas propriedades, é possível a introdução de uma tema muito importante da geometria do ensino básico que são os quadriláteros e suas propriedades. Com o uso correto dessas ferramentas, o professor pode explorar, por meio da tecnologia, conceitos básicos, como a classificação dos quadriláteros.

Além disso, a escolha da atividade ocorreu para que os professores pudessem aprender a criar uma nova ferramenta no software GeoGebra, 
denominada macro e, para isso, deveriam selecionar a opção Criar uma nova Ferramenta, como ilustrado na Figura 2.

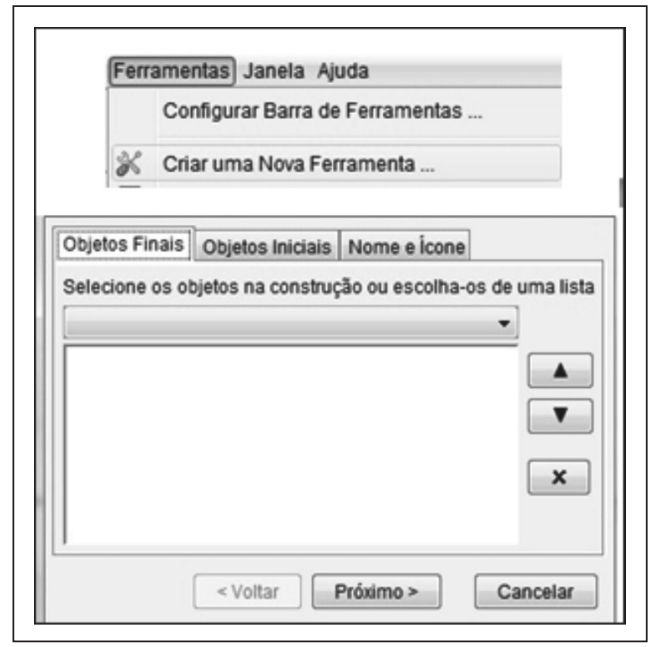

Figura 2 - Caminho necessário para criar uma nova ferramenta no GeoGebra

Seguindo as orientações dadas, os professores participantes deveriam explorar a janela de criação de uma nova ferramenta (Figura 2), de tal forma a atingir o objetivo dado, ou seja, ao selecionar dois pontos quaisquer da tela (objetos iniciais), o resultado deveria ser um retângulo (objeto final) que possui esses dois pontos como dois de seus vértices.

Para a prática do professor, o uso de macros pode colaborar no planejamento de aulas e na execução de atividades propostas aos alunos, eliminando etapas consideradas desnecessárias. Um exemplo disso pode ser uma atividade de classificação de retângulos, pois com a construção de uma ferramenta macro que dê como resposta um retângulo, é possível a construção de vários retângulos com poucos cliques na tela do software GeoGebra.

$\mathrm{O}$ procedimento de uso de macros pode permitir que o professor se aprofunde na discussão com seus alunos sobre os resultados apresentados, uma vez que os mesmos não fariam o passo a passo de construção de retângulos, cada vez que fossem utilizados, poupando um tempo que pode ser precioso para o desenvolvimento da atividade.

De forma geral, os temas das seis lições foram escolhidos para que os professores participantes pudessem explorar tanto as ferramentas geométricas quanto as ferramentas algébricas disponíveis no software GeoGebra. 
É importante notar que essa integração entre as ferramentas geométricas e as ferramentas algébricas possibilita o desenvolvimento das atividades em vários registros de representações dos objetos matemáticos.

\section{$6 \mathrm{O}$ desenvolvimento e análise de três atividades propostas nas oficinas}

O objetivo da atividade I era que os professores, durante a oficina, nos seus primeiros contatos com o GeoGebra, conhecessem o seu menu, utilizassem algumas ferramentas, fizessem uso do campo entrada do GeoGebra e, também, gravassem um arquivo. Além disso, a atividade I propicia que os professores entendam a relação entre as diferentes janelas do GeoGebra: visualização e álgebra. Para isso, a atividade sugere que os professores construam um triângulo qualquer, meçam seus ângulos e verifiquem o que acontece na janela algébrica com a movimentação de qualquer vértice.

Apesar de ser uma atividade simples, o professor, nesse primeiro contato com o GeoGebra, teve acesso a uma das principais características do software, que é trabalhar ao mesmo tempo com mais de uma forma de registro de representação de um mesmo objeto matemático, fato que facilita a compreensão em Matemática.

Ao relacionar a movimentação dos vértices do triângulo com as alterações da janela de álgebra, e chegando à conclusão de que a soma dos ângulos internos não é alterada, inicia-se o trabalho com uma das dimensões da Gênese Instrumental proposta por Rabardel (1995), que é a instrumentação. Isso porque o professor precisa relacionar seu conhecimento anterior sobre triângulos com as relações que o software apresenta, verificando e testando suas hipóteses que, no caso específico dessa atividade, trata-se da soma dos ângulos internos de um triângulo e sua não-variação, independente do triângulo apresentado pelo software e das movimentações realizadas pelo usuário.

Para perceber as relações entre as janelas de visualização e de álgebra, os professores deveriam observar as alterações ocorridas na janela de álgebra com a movimentação de um dos vértices do triângulo. Um caso dessa relação pode ser observado na Figura 3. 


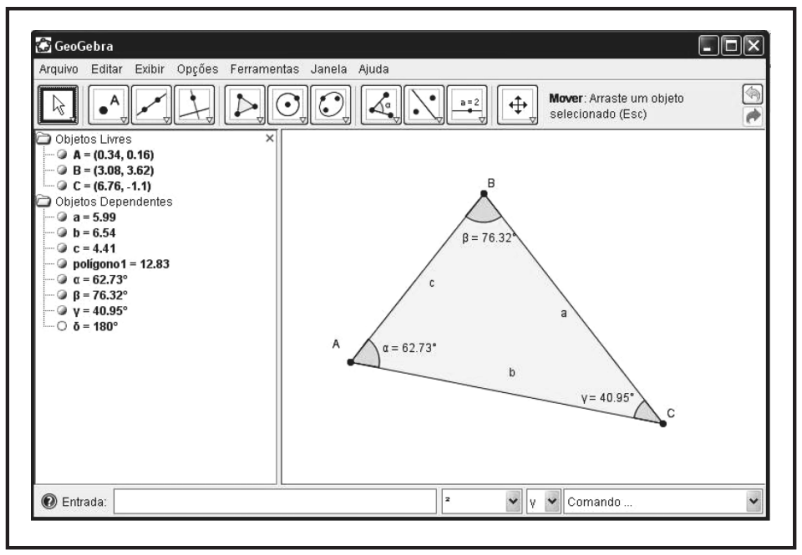

Figura 3 - Relação entre as janelas de visualização e de álgebra (software GeoGebra)

As dificuldades apresentadas durante a atividade I foram pontuais, mas nada referente ao software GeoGebra ou aos entes matemáticos explorados. Tais dificuldades foram concentradas em como localizar, por exemplo, a pasta correta para salvar os arquivos.

Dessa forma, como a dificuldade não estava relacionada com o software GeoGebra e nem com a Matemática, descartando qualquer obstáculo relacionado às variáveis do Design Experiments, a atividade I permaneceu com a mesma estrutura nas três oficinas realizadas.

Além disso, pode-se verificar sucesso nas várias interações que ocorrem nas atividades instrumentais, denominadas por Rabardel (1995) como modelo SAI (Figura 1):

-A relação sujeito-objeto: os professores conseguiram observar de forma eficaz as ações transformadoras dirigidas sobre o objeto que, no caso, era o triângulo construído com o software GeoGebra, por meio das mudanças simultâneas que ocorreram entre as características geométricas do triângulo (desenho apresentado na tela) e as características algébricas do triângulo (medidas de ângulos e sua soma, apresentados na janela algébrica).

- A relação sujeito-instrumento: os professores conseguiram realizar todo o processo de construção e manipulação do objeto (triângulo), utilizando as ferramentas e os resultados fornecidos pelo instrumento (software GeoGebra), para chegar às conclusões sobre a soma dos ângulos internos do triângulo. 
- A relação instrumento-objeto: o instrumento (software GeoGebra) atendeu às necessidades de construção e manipulação do objeto (triângulo).

- A relação sujeito-objeto mediada pelo instrumento: consequência da boa realização das demais interações.

Por meio desses resultados, a atividade I foi desenvolvida de forma satisfatória no ambiente formado pelo conjunto de condições que o sujeito deve levar em conta para realizar sua atividade, que é o modelo SAI de Rabardel (1995). Além disso, considerando as variáveis dependentes do Design Experiments, a análise da atividade I permitiu uma direção para o processo de desenvolvimento das demais atividades.

Dessa forma, os esquemas de uso, que são classificados por Rabardel (1995) como as atividades relacionadas ao funcionamento e manipulação, neste caso, do GeoGebra, foram desenvolvidos com êxito pelos professores, caracterizando, então, a ocorrência do processo de instrumentalização da Gênese Instrumental. Os esquemas de ação instrumental, que são, de acordo com Rabardel (1995), orientados ao objeto da atividade (nesse caso o triângulo) no qual o artefato (o software GeoGebra) é um meio de concretização e de realização, também foram desenvolvidos com êxito pelos professores o que caracteriza a ocorrência do processo de instrumentação da Gênese Instrumental.

Observou-se também, durante a realização da atividade I e em todas as oficinas aplicadas, que os professores sempre estavam dialogando entre eles, com o objetivo de partilhar ideias e hipóteses. Surgiram, então, os esquemas de atividade coletiva instrumental, caracterizados, por Rabardel (1995), como a inserção dos sujeitos em uma atividade coletiva de esquemas de utilização, que apresentam a coordenação de ações individuais e a integração de seus resultados para atender aos objetivos comuns.

Para a construção do triângulo equilátero, na Atividade II, os professores foram orientados a configurar a caixa de ferramentas do GeoGebra, deixando visíveis apenas as ferramentas necessárias.

O objetivo dessa configuração foi inserir os professores na dimensão da instrumentalização da Gênese Instrumental de Rabardel (1995), caracterizada como um processo pelo qual o sujeito personaliza o artefato de acordo com as suas necessidades. É importante observar que, ao limitarmos o uso de ferramentas, exige-se do professor o conhecimento matemático de triângulo equilátero e suas etapas de construção.

Na primeira oficina, apenas um dos professores não configurou de forma 
correta a caixa de ferramentas. Por se tratar de um caso isolado, essa ocorrência não afetou a segunda nem a terceira oficina, visto que todos os professores configuraram de forma correta a caixa de ferramentas.

Seguindo na mesma atividade, os professores deveriam construir um triângulo eqüilátero, utilizando apenas as ferramentas disponíveis, de acordo com a configuração solicitada. O objetivo da atividade foi inserir os professores na dimensão da instrumentação da Gênese Instrumental de Rabardel (1995), que é o processo pelo qual as ações do sujeito para resolver um dado problema são condicionadas pelas especificidades e potencialidades de um artefato.

Levando-se em consideração as propriedades matemáticas e as ferramentas disponíveis, uma das possíveis soluções para a construção de um triângulo equilátero seria a construção de um dos lados do triângulo e sua mediatriz, sendo que qualquer uma das interseções das circunferências, de raio de medida do segmento $\mathrm{AB}$, utilizadas para a construção da mediatriz determina o terceiro vértice do triângulo. Tal construção garante que o triângulo é equilátero porque os lados são congruentes, uma vez que os raios das circunferências possuem a mesma medida do segmento com extremos em A e B.

Na primeira oficina, dois professores não construíram o triângulo equilátero de forma correta. Um deles fez o processo de construção de forma correta, mas, no momento de clicar para a construção das circunferências, o fez fora do ponto B. A outra construção incorreta do triângulo equilátero foi feita por aproximação.

Essas construções incorretas do triângulo equilátero estão inseridas nas variáveis de aprendizagem do Design Experiments. Portanto, para tentar evitar construções descritas anteriormente, na segunda oficina foi acrescentada uma figura representando um esboço da construção e, na terceira oficina, acrescentouse a definição de triângulo equilátero. Tais procedimentos evidenciam que outras orientações direcionadas para o conteúdo matemático foram necessárias para que os professores realizassem a construção do triângulo equilátero de forma correta, utilizando o software GeoGebra.

De forma geral, assim como ocorreu na atividade I, pode-se verificar sucesso nas várias interações que ocorreram com o software Geogebra nas atividades instrumentais.

Por meio desses resultados, a atividade II, assim como a atividade I, foi desenvolvida de maneira satisfatória no ambiente formado pelo conjunto de condições que se deve levar em conta para sua realização, que é o modelo SAI de Rabardel (1995). 
Dessa forma, os esquemas de uso (RABARDEL, 1995) foram desenvolvidos com êxito pelos professores, caracterizando, então, a ocorrência do processo de instrumentalização da Gênese Instrumental, uma vez que a configuração e uso das caixas de ferramentas ocorreram corretamente. Os esquemas de ação instrumental (RABARDEL, 1995) também foram desenvolvidos com êxito pelos professores, fato que caracteriza a ocorrência do processo de instrumentação da Gênese Instrumental.

\section{Considerações finais}

Os resultados dos dois primeiros encontros permitiram uma análise que possibilitou o aprimoramento do terceiro encontro com os professores.

Em relação à Gênese Instrumental, verificou-se que a maioria dos professores conseguiu alcançar o processo de instrumentalização, uma vez que, por exemplo, localizaram corretamente as ferramentas necessárias para a construção do triângulo equilátero, configurando o ambiente GeoGebra para isso. Também verificou-se que a característica dinâmica do software GeoGebra colaborou para a ocorrência da instrumentação, uma vez que, ao manipular as potencialidades do software, os professores condicionaram suas ações para responder aos questionamentos realizados.

Como a metodologia utilizada foi o Design Experiments, algumas mudanças na realização e estrutura de cada oficina foram efetuadas, por meio da análise prévia. Tais ações tiveram como objetivo levar o professor à melhor reflexão no momento de analisar a construção realizada no GeoGebra e, consequentemente, procurar um embasamento adequado para responder aos questionamentos propostos nas atividades.

\section{Referências}

BRASIL. Ministério da Educação e do Desporto: Parâmetros Curriculares Nacionais: Introdução. Ensino de quinta a oitava série. Brasília: Ministério da Educação, 1997.

COBB, P.; CONFREY, P.; DISESSA, A.; LEHRER, R.; SCHAUBLE, L. Design experiments in educational research. Educational Researcher, Washington, DC, v. 32, n. 1, p. $9-13$, Jan./Feb. 2003. 
COLLINS, A; JOSEPH, D; BIELACZYC, K. Design Research: Theoretical and Methodological Issues. Journal of The Learning Sciences, Evanston, v. 13, n. 1, p. 13-42, Jan. 2004.

DOERR, H. M.; WOOD, T. Pesquisa-Projeto (design research): aprendendo a ensinar Matemática. In: BORBA, M. C. (Org.). Tendências internacionais em formação de professores de matemática. Belo Horizonte: Autêntica, 2006. p. 113-128.

LABORDE C.; STRÄßER, R. Place and use of new technology in the teaching of mathematics: ICMI activities in the past 25 years. ZDM Mathematics Education, Eggenstein-Leopoldshafen, Alemanha, DE, v.42, n.1, p.121-133, Feb. 2010.

RABARDEL, P. Les hommes et les technologies: une approche cognitive des instruments contemporains. Paris: Armand Colin, 1995.

RABARDEL, P.; WAERN, Y. From artefact to instrument. Interacting with Computers, Linkojing, Suécia, v. 15, n. 5, p. 641-645, 2003.

SALAZAR, J. V. F. Gênese instrumental na interação com Cabri 3D: um estudo de transformações geométricas no espaço. 2009, 319f, Tese (Doutorado em Educação Matemática) - Programa de Estudos Pós Graduados em Educação Matemática. Pontifícia Universidade Católica de São Paulo, São Paulo, 2009. Disponível em: <http:/ /www.pucsp.br/pos/edmat>. Acesso em: 07 mar. 2013.

VERGNAUD, G. A teoria dos campos conceptuais. In: Jean Brun (Ed.) Didáctica das Matemáticas. Lisboa: Instituto Piaget, 1996.

ZUCHI, I. A integração dos ambientes tecnológicos em sala: novas potencialidades e novas formas de trabalho. In: SIMPÓSIO INTERNACIONAL DE PESQUISA EM EDUCAÇÃO MATEMÁTICA, 2, 2008, Recife. Matemática Formal e Matemática nãoFormal 20 anos depois: sala de aula e outros contextos. Universidade Federal Rural de Pernambuco, 155f, Recife, 2008. Disponível em: http://www.ded.ufrpe.br/ sistemática/CD-Rom\%202\%2OSIPEMAT/artigos/CO-167.pdf> Acesso em: 07 mar. 2013.

Submetido em Agosto de 2012. Aprovado em Janeiro de 2013. 
